\title{
TLR4 signaling induces TLR2 expression in endothelial cells via neutrophil NADPH oxidase
}

\author{
Jie Fan, Randall S. Frey, and Asrar B. Malik \\ Department of Pharmacology, University of Illinois College of Medicine, Chicago, Illinois, USA
}

\begin{abstract}
Interactions of polymorphonuclear neutrophils (PMNs) with endothelial cells may contribute to the activation of endothelial cell responses involved in innate immunity. We explored a novel function of PMN NADPH oxidase in the mechanism of Toll-like receptor-2 (TLR2) upregulation induced by LPSTLR4 signaling in endothelial cells. We showed that LPS induced TLR2 up-regulation through TLR4and MyD88-dependent signaling. In neutropenic mice, the LPS-induced NF-kB activation and TLR2 expression were significantly reduced, and both responses were restored upon repletion by PMN obtained from WT mice but not by PMNs from NADPH oxidase $g p^{9} 1^{p h o-/}$ mice. These findings were recapitulated in mouse lung vascular endothelial cells cocultured with PMNs, indicating that the augmented NF-kB activation and the resultant TLR2 upregulation in endothelial cells were secondary to oxidant signaling generated by PMN NADPH oxidase. The functional relevance of NADPH oxidase in mediating TLR4-induced TLR2 expression in endothelial cells was evident by markedly elevated and stable ICAM-1 expression as well as augmented PMN migration in response to sequential challenge with LPS and peptidoglycan. Thus, PMN NADPH oxidase-derived oxidant signaling is an important determinant of the cross talk between TLR 4 and TLR 2 and the control of endothelial cell activation.

J. Clin. Invest. 112:1234-1243 (2003). doi:10.1172/JCI200318696.
\end{abstract}

\begin{abstract}
Introduction
The vascular endothelium is a multifunctional cell monolayer that plays important roles in the regulation of vascular tone, coagulation and fibrinolysis, cellular growth, differentiation, and immune and inflammatory responses (1-3). For example, infection caused by invasion of microbial pathogens in the lungs leads to the activation of the first-line host-defense mechanisms, which include antimicrobial molecules and phagocyte defenses provided by resident alveolar macrophages and polymorphonuclear neutrophils (PMNs) recruited into the affected parenchyma (4). Vascular endothelial cells play a critical role in regulating PMN migration by production of proinflammatory molecules, including leukocyte adhesion proteins, cytokines, and chemokines (5-7).
\end{abstract}

Received for publication April 18, 2003, and accepted in revised form August 19, 2003.

Address correspondence to: Asrar B. Malik, Department of Pharmacology, University of Illinois College of Medicine, 835 South Wolcott Avenue, Room E425, Chicago, Illinois 60612, USA. Phone: (312) 996-7635; Fax: (312) 996-1225;

E-mail:abmalik@uic.edu.

Jie Fan's present address is: Department of Surgery,

School of Medicine, University of Pittsburgh, Pittsburgh,

Pennsylvania, USA.

Conflict of interest: The authors have declared that no conflict of interest exists.

Nonstandard abbreviations used: polymorphonuclear neutrophil (PMN); Toll-like receptor (TLR); peptidoglycan (PGN); mouse lung vascular endothelial cell (MLVEC); electrophoretic mobility shift assay (EMSA); bronchoalveolar lavage fluid (BALF).
As a part of the innate immune response, pattern recognition receptors mediate the interaction between conserved patterns on microorganisms, pathogen-associated molecular patterns, and the host. Toll-like receptors (TLRs) have been demonstrated as pathogenassociated molecular pattern receptors for diverse microbial-derived molecules. To date, ten TLR family members have been identified in the human genome. TLR2 is crucial for the propagation of the inflammatory response to components of gram-positive and gram-negative bacteria and mycobacteria such as peptidoglycan (PGN), lipoteichoic acid, bacterial lipoproteins, lipopeptides, and lipoarabinomannan (8-11). TLR2 is predominantly expressed in the cells involved in first-line host defense, including monocytes, macrophages, dendritic cells, and PMNs (12-15). A lower expression level was observed in endothelial and epithelial cells (16). TLR4 has been identified as the receptor for LPS and lipoteichoic acid $(17,18)$. Recently, we reported an important function of TLR4 signaling in regulating PMN migration by modulation of the cell surface expression of chemokine receptors (19), indicating that cross talk between TLR4 and G protein-coupled receptors is a critical determinant of the host-defense response. It has been reported that LPS may induce TLR2 expression in human endothelial cells in an NF-KB-dependent manner (20), suggesting a role of NF-KB in the possible cross talk between TLR 2 and TLR 4 signaling.

NF-kB signaling occurs via an oxidant-sensitive pathway (21-25). Our previous studies showed that the endothelial cell is a key target of PMN NADPH oxidase activity (26). PMN NADPH oxidase-derived oxidants mediate the 
early-onset TNF- $\alpha$-induced NF- $\kappa \mathrm{B}$ activation and ICAM-1 expression in endothelial cells (26). Although LPS induces oxidative burst in PMNs $(27,28)$, it remains unclear whether the released oxidants contribute to the LPS-induced NF-KB activation and thus are involved in signaling TLR4-TLR2 cross talk in the endothelium.

PMN NADPH oxidase consists of five subunits: $\mathrm{p} 40^{\text {phox }}, \mathrm{p} 47^{\text {phox }}, \mathrm{p} 67^{\text {phox }}, \mathrm{p} 22^{\text {phox }}$, and gp91 ${ }^{\text {phox }}$. In the basal state, $\mathrm{p} 40^{\text {phox }}$, $\mathrm{p} 47^{\text {phox }}$, and $\mathrm{p} 67^{\text {phox }}$ exist in the cytosol as a complex, while p22phox and gp91 phox are located in membranes of secretory vesicles and specific granules of PMNs, where they are present as a heterodimeric flavohemoprotein known as cytochrome $b_{558}(29)$. Two low-molecular-weight GTP-binding proteins, Rap1A and Rac1/2, are also involved in the activation of NADPH oxidase (29). The cytosolic component $\mathrm{p} 47^{\text {phox }}$ is phosphorylated upon stimulation, and the entire cytosolic complex migrates to the membrane, where it associates with cytochrome $b_{558}$ to assemble the active oxidase (29).

In the present study, we addressed the role of the PMN NADPH oxidase complex in mediating the cross talk between TLR4 and TLR2. We used cocultures of normal mouse lung vascular endothelial cells (MLVECs) and PMNs genetically deficient in the gp91phox or p47phox subunit of NADPH oxidase. We demonstrate here that the functional impairment of PMN NADPH oxidase, and thereby of oxidant generation, markedly decreased the LPS-induced NF-KB activation and TLR2 upregulation in MLVECs and lungs. Using TLR4 knockout and MyD88 knockout mice, we establish that the LPS upregulation of TLR2 is both TLR4- and MyD88-dependent. The results define the functional relevance of PMN NADPH oxidase in mediating TLR2 upregulation in endothelial cells that in turn activates the augmented ICAM- 1 expression in response to microbial products. Thus, TLR2 activation, signaled by TLR 4 and as regulated by PMN NADPH oxidase, is an important mechanism responsible for amplifying PMN transmigration to sites of infection.

\section{Methods}

Mice. Mice deficient in gp9 $91^{\text {phox }}$ were obtained from Mary Dinauer (University of Indiana School of Medicine, Indianapolis, Indiana, USA) (30). Breeder stocks for $p 47^{p h o x-1-}$ mice were obtained from Steven Holland (Laboratory of Host Defenses, National Institute of Allergy and Infectious Diseases, NIH, Bethesda, Mary-

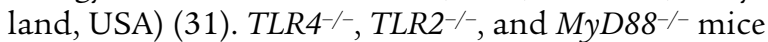
were obtained from Shizuo Akira (Research Institute for Microbial Diseases, Osaka University, Osaka, Japan) (32-34). WT mice of similar genetic background (C57BL/6) were purchased from The Jackson Laboratory (Bar Harbor, Maine, USA). All animals were maintained under specific pathogen-free conditions in a barrier facility. Mice were 9-10 weeks of age at the time of experiments. All animal studies were made in compliance with the appropriate University of Illinois institutional review board.
Experimental protocols. Animals were given LPS (10 $\mu \mathrm{g} / 10 \mathrm{~g}$ body wt; Sigma-Aldrich, St. Louis, Missouri, USA), live E. coli $\left(1 \times 10^{5}\right.$ cells $/ 10 \mathrm{~g}$ body wt), or saline by intraperitoneal injection, bronchoalveolar lavage fluid was collected, and whole lungs were then harvested. In some experiments, neutropenia was induced by PMN depletion using rabbit anti-mouse neutrophil serum (Intercell Technologies, Hopewell, New Jersey, USA). At approximately 16 hours before injection of LPS, $50 \mu \mathrm{l}$ of ANS or control antibody (rabbit anti-mouse IgG; Sigma-Aldrich) was administered intraperitoneally to mice. Blood for determination of absolute PMN counts was obtained by cardiac puncture from animals that had received ANS or control serum. PMN counts were determined manually on Wright-Giemsa-stained slides prepared with Cytospin centrifuge (Shandon Inc., Pittsburgh, Pennsylvania, USA). On average, the PMN count in the ANS group was decreased to $0.08 \% \pm 0.02 \%$ of total white blood cells compared with $22.2 \% \pm 1.9 \%$ in the control group. There were no statistically significant differences in the number of peripheral lymphocytes, monocytes, atypical lymphocytes, or eosinophils between the ANS and control groups.

To determine the role of PMN NADPH oxidase in the mechanism of TLR4-induced TLR2 expression, we performed PMN repletion experiments in neutropenic mice using PMNs $\left(\sim 1 \times 10^{6}\right.$ cells $)$ isolated from multiple WT or $g p 91^{\text {phox }-/-}$ mice. PMN isolation was carried out as described (35) using NIM-2 leukocyte isolation medium (Cardinal Associates, Santa Fe, New Mexico, USA).

MLVEC isolation and characterization. MLVECs were isolated using a modification of the described method (36). Briefly, mice anesthetized with $3 \%$ halothane and heparin $(50 \mathrm{U})$ were injected into the jugular vein as an anticoagulant. The abdominal cavity was opened and the pulmonary artery was cannulated. Krebs-Henseleit solution supplemented with BSA $(5 \mathrm{~g} / 100 \mathrm{ml})$ was infused to remove blood from lungs. Peripheral lung tissue slices were prepared, washed, and suspended in HBSS. The tissue slices were minced and digested with collagenase A $(1.6 \mathrm{mg} / \mathrm{ml}$ in HBSS $)$ for 20 minutes at $37^{\circ} \mathrm{C}$ in a shaking water bath. The released cells were centrifuged and suspended in $5 \mathrm{ml}$ suspension buffer $\left(\mathrm{Ca}^{2+}\right.$-free and $\mathrm{Mg}^{2+}$-free PBS containing $0.5 \mathrm{~g} / 100 \mathrm{ml}$ BSA, $2 \mathrm{mM}$ EDTA, and $4.5 \mathrm{mg} / \mathrm{ml}$ D-glucose) and filtered through a $200-\mu \mathrm{m}$ mesh filter followed by $60-\mu \mathrm{m}$ mesh. The filtered cells were washed and finally suspended in $8 \mathrm{ml}$ of growth medium (MEM D-Val medium containing $2 \mathrm{mM}$ glutamine, $10 \% \mathrm{FBS}, 5 \%$ human serum, $50 \mu \mathrm{g} / \mathrm{ml}$ penicillin/streptomycin, $5 \mu \mathrm{g} / \mathrm{ml}$ heparin, $1 \mu \mathrm{g} / \mathrm{ml}$ hydrocortisone, $5 \mu \mathrm{g} / \mathrm{ml}$ endothelial cell growth supplement from bovine brain, $5 \mu \mathrm{g} / \mathrm{ml}$ amphotericin, and $5 \mu \mathrm{g} / \mathrm{ml}$ mycoplasma removal agent). The cells were then transferred to a $100-\mathrm{mm}$ culture dish. The cells were allowed to grow for 3-4 days. The endothelial cells (which appeared in patches) were identified by light microscopy. Cloning rings were placed on these cells, and the cells were 
trypsinized and transferred to culture dishes. The cells were characterized by their cobblestone morphology, uptake of Dil-Ac-LDL (Biomedical Technologies Inc., Stoughton, Massachusetts, USA), and factor VIIIrelated antigen staining (Sigma-Aldrich). After 2-3 days, when cells were confluent, they were washed twice with PBS and the medium was changed to lowserum medium (1\% FBS). Cells were treated with repurified LPS $(1 \mu \mathrm{g} / \mathrm{ml})$ and/or cocultured with PMNs $\left(1 \times 10^{5}\right.$ cells $\left./ \mathrm{ml}\right)$ that had been isolated from WT, $p 47^{\text {phox-l-- }}$, or $g p^{91^{\text {phox }}-/-}$ mice. In some experiments, to further address the role of PMN-derived oxidants in the mechanism of LPS-induced TLR2 upregulation in endothelial cells, PEG-catalase $(1,000 \mathrm{U} / \mathrm{ml}$, SigmaAldrich) was added to the coculture system. The cells were incubated with PEG-catalase for 2 hours and then harvested for Western analysis.

Western blot analysis. Lung tissue homogenate samples or aliquots of MLVEC lysate were separated by $10 \%$ SDS-PAGE under nonreducing conditions. Equivalent loading of the gel was determined by quantification of protein as well as reprobing membranes for actin detection. Separated proteins were electroblotted onto PVDF membrane and blocked for 1 hour at room temperature with Tris-buffered saline containing $1 \%$ BSA. The membranes were then probed with a 1:1,000 dilution of purified polyclonal IgG against mouse TLR2 (Santa Cruz Biotechnology Inc., Santa Cruz, California, USA) at room temperature for 1 hour. After washing, primary antibodies associated with the membranes were detected on autoradiographic film by HRP-conjugated secondary antibodies and the ECL Plus chemiluminescence system (Amersham Biosciences, Arlington Heights, Illinois, USA) according to the manufacturer's instructions.

RT-PCR. Total RNA from lungs or MLVECs was isolated using TRI Reagent (Molecular Research Center, Cincinnati, Ohio, USA) following the manufacturer's instructions. Total RNA was then reverse-transcribed using a SuperScript Preamplification kit (Invitrogen Corp., Carlsbad, California, USA). Primers for TLR2 amplification were: position 1,008 forward, $5^{\prime}$-ATACTAACTTGGCCAGGTTC-3'; position 1,590 reverse, $5^{\prime}$ AAAGTGTTCCTGCTGATGTC-3', amplifying $582 \mathrm{bp}$. Reverse transcription product was amplified following the kit instructions. PCR products were separated using $1.2 \%$ agarose gel and identified by ethidium bromide staining.

Nuclear protein extraction. Nuclear protein extracts were prepared from lung tissue or MLVECs by the method of Deryckere and Gannon (37). Aliquots of $100 \mathrm{mg}$ of frozen lung tissue were ground to powder with a mortar in liquid nitrogen. The thawed powder or $1 \times 10^{7}$ cells of MLVEC were homogenized in a Dounce tissue homogenizer with $4 \mathrm{ml}$ of a solution consisting of $0.6 \%$ Nonidet P- $40,150 \mathrm{mM} \mathrm{NaCl}, 10$ mM HEPES ( $\mathrm{pH} 7.9$ ), $1 \mathrm{mM}$ EDTA, and $0.5 \mathrm{mM}$ PMSF. The cells were lysed with five strokes of the pestle. After transfer to a $15-\mathrm{ml}$ tube, the debris was pelleted by centrifuging at $600 \mathrm{~g}$ for 30 seconds. The supernatant was transferred to $50-\mathrm{ml}$ tubes, incubated on ice for 5 minutes, and centrifuged for $10 \mathrm{~min}$ utes at $3,600 \mathrm{~g}$. Nuclear pellets were then resuspended in $300 \mu \mathrm{l}$ of a solution consisting of $25 \%$ glycerol, $20 \mathrm{mM}$ HEPES at $\mathrm{pH} 7.9,420 \mathrm{mM} \mathrm{NaCl}, 1.2 \mathrm{mM}$ $\mathrm{MgCl}_{2}, 0.2 \mathrm{mM}$ EDTA, $0.5 \mathrm{mM}$ DTT, $0.5 \mathrm{mM}$ PMSF, $2 \mathrm{mM}$ benzamidine, $5 \mu \mathrm{g} / \mathrm{ml}$ pepstatin, $5 \mu \mathrm{g} / \mathrm{ml}$ leupeptin, and $5 \mu \mathrm{g} / \mathrm{ml}$ aprotinin and incubated on ice for 20 minutes. The mixture was transferred to microcentrifuge tubes, and nuclei were pelleted by centrifugation at $16,000 \mathrm{~g}$ for 1 minute. Supernatants containing nuclear proteins were frozen in liquid nitrogen in small aliquots and stored at $-70^{\circ} \mathrm{C}$. Protein quantification was performed using Bio-Rad Protein Assay Dye Reagent (Bio-Rad Laboratories, Hercules, California, USA).

Electrophoretic mobility shift assay. The probe for electrophoretic mobility shift assay (EMSA) is a 24-bp double-stranded construct of NF- $\mathrm{\kappa B}$ consensus binding sequence $\left(5^{\prime}\right.$-AGGGACTTTCCGCTGGGACTTTCC$\left.3^{\prime}\right)$. End labeling was performed by T4 kinase in the presence of $\left[{ }^{32} \mathrm{P}\right]$ ATP. Labeled oligonucleotides were purified on a Sephadex G-50 M column (Amersham Biosciences, Piscataway, New Jersey, USA). An aliquot of $5 \mu \mathrm{g}$ of nuclear protein was incubated with the labeled double-stranded probe $(\sim 50,000 \mathrm{cpm})$ in the presence of $5 \mu \mathrm{g}$ of the nonspecific blocker poly(dI$\mathrm{dC})$ in binding buffer (10 mM Tris-HCl, $\mathrm{pH} 7.5,100$ $\mathrm{mM} \mathrm{NaCl}, 1 \mathrm{mM}$ EDTA, $0.2 \%$ Nonidet P-40, and 0.5 $\mathrm{mM}$ DTT) at $25^{\circ} \mathrm{C}$ for 20 minutes. Specific competition was performed by adding $100 \mathrm{ng}$ of unlabeled double-stranded oligonucleotide, while for nonspecific competition, $100 \mathrm{ng}$ of unlabeled double-stranded mutant oligonucleotide (5'-AGCTCAATCTCCCTGGGACTTTCC-3'), which does not bind NF- $\kappa B$, was added. The mixture was separated by electrophoresis on a $5 \%$ polyacrylamide gel in $1 \times$ Tris glycine EDTA buffer. Gels were vacuum-dried and subjected to autoradiography and Fuji BAS2500 phosphoimager (Fujifilm, Stamford, Connecticut, USA) analysis.

Repurification of LPS. Repurification of LPS was carried out using a described method $(38,39)$. In brief, $5 \mathrm{mg}$ of LPS was resuspended in $1 \mathrm{ml}$ of endotoxin-free water containing $0.2 \%$ triethylamine. Deoxycholate was added to a final concentration of $0.5 \%$, followed by the addition of $500 \mu$ l of water-saturated phenol. The sample was vortexed intermittently for 5 minutes, and the phases were allowed to separate at room temperature for 5 minutes. The sample was placed on ice for $5 \mathrm{~min}$ utes, followed by centrifugation at $4^{\circ} \mathrm{C}$ for 2 minutes at $10,000 \mathrm{~g}$. The top aqueous layer was re-extracted with $1 \mathrm{ml}$ of water-saturated phenol, and the phenol phase was subjected to re-extraction with $500 \mu \mathrm{l}$ of $0.2 \%$ triethylamine $/ 0.5 \%$ deoxycholate. The aqueous phase was adjusted to $75 \%$ ethanol and $30 \mathrm{mM}$ sodium acetate and was allowed to precipitate at $-20^{\circ} \mathrm{C}$ for 1 hour. The precipitates were centrifuged at $4^{\circ} \mathrm{C}$ for 10 minutes at 10,000 $g$, washed in $1 \mathrm{ml}$ of cold 100\% ethanol, and air-dried. The 
$\mathbf{a}$

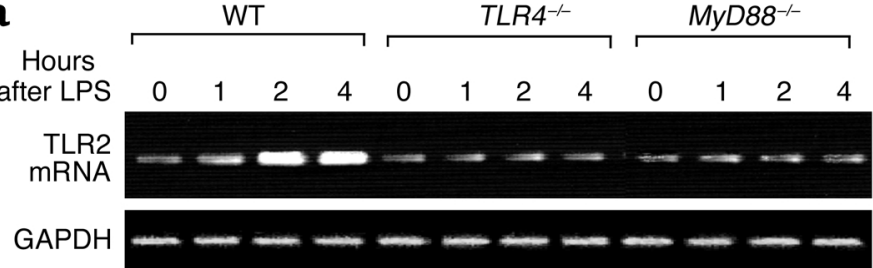

b

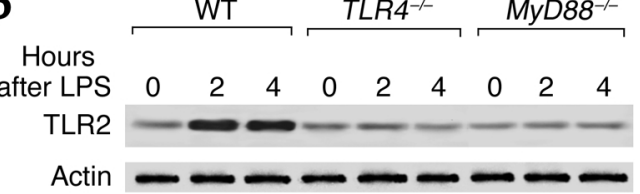

C

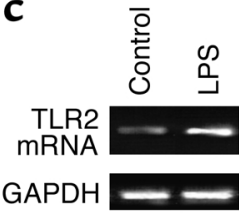

d

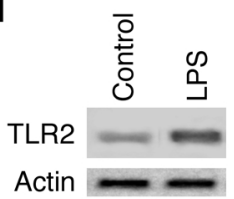

\section{Figure 1}

LPS upregulates TLR2 expression in lungs and endothelial cells through TLR4 and MyD88 signaling. (a and $\mathbf{b})$ Mice were injected intraperitoneally with LPS $(10 \mu \mathrm{g} / 10 \mathrm{~g}$ body wt) and whole lungs were harvested at the times indicated after LPS injection. Data are representative of three independent studies. (a) RT-PCR for TLR2 mRNA expression in the lungs. GAPDH mRNA is identified as a control for RNA loading. (b) Western blot for TLR2 protein in lungs. Corresponding actin is shown as evidence of comparable loading between lanes. (c and d) MLVECs were incubated with LPS $(1 \mu \mathrm{g} / \mathrm{ml})$ for 2 hours, TLR2 mRNA was detected using RT-PCR (c), and TLR2 protein level was measured by Western blotting $(\mathbf{d})$. The data are representative of three independent studies.

MyD88 deficiency prevented LPS upregulation of TLR2. The changes in protein expression of TLR2 shown in Figure $1 \mathrm{~b}$ paralleled the changes in mRNA.

To address whether endothelial cells were responsible for the changes in LPSinduced TLR2 expression, MLVECs were stimulated with LPS in vitro, and TLR2 mRNA and protein were assessed. LPS caused the upregulation of TLR2 expression in MLVECs at 2 hours (Figure 1, c and d), consistent with the changes seen in lung tissue.

$N F-\kappa B$ mediates LPSTLR4-induced upregulation of TLR2. The promoter region of the TLR2 gene contains

precipitates were resuspended in the original volume $(500 \mu \mathrm{l})$ of $0.2 \%$ triethylamine. Recovery of $100 \%$ was assumed for the purified LPS samples (38).

In vivo PMN migration assay. Mouse air pouches were prepared as described (40). We first challenged the mice with LPS $(10 \mu \mathrm{g} / 10 \mathrm{~g}$ body wt intraperitoneally in $0.5 \mathrm{ml}$ of saline) or saline. The chemokine MIP- 2 was injected 1 hour later into each mouse's air pouch. This was followed 1 hour later (or 2 hours after the initial challenge) by a second dose of either LPS $(10 \mu \mathrm{g} / 10 \mathrm{~g}$ body wt intraperitoneally in $0.5 \mathrm{ml}$ of saline) or PGN $(10 \mu \mathrm{g} / 10 \mathrm{~g}$ body wt intraperitoneally in $0.5 \mathrm{ml}$ of saline). The pouch lavage fluid was collected for PMN counts at 2 hours and 8 hours after the first injection of LPS or saline.

Statistics. The data are presented as mean \pm SEM of the indicated number of experiments. Statistical significance among group means was assessed by ANOVA. The Student Newman-Keuls post-hoc test was performed. Differences were considered significant at $P<0.05$.

\section{Results}

TLR4 signaling upregulates TLR2 expression in lungs and MLVECs. As shown in Figure 1a, LPS challenge of WT mice caused an increase in TLR 2 mRNA expression at 1 hour, and marked increases at 2 hours and 4 hours. However, in TLR4 $4^{-/-}$mice, LPS failed to induce TLR2 expression (Figure 1a), indicating that TLR4 signaling mediates the LPS-induced upregulation of TLR2. MyD88 is a signaling adapter containing the Toll/IL-1R domain, the conserved motif essential for TLR signal transduction (41). Previous studies showed that TLR4 can signal through both MyD88-dependent and -independent pathways (41). To address the role of MyD88 in mediating LPS-TLR4-induced upregulation of TLR2, LPS was injected into $M y D 88^{-/-}$mice and lung TLR2 mRNA expression was assessed. As shown in Figure 1a, two NF- $\kappa \mathrm{B}$ consensus binding sequences believed to regulate gene transcription (42). We carried out a gelshift assay using extracts from whole lung tissue to address whether LPS-TLR4 upregulation of TLR2 was the result of activation of NF- $\kappa \mathrm{B}$. As shown in Figure $2 \mathrm{a}$, the response to LPS in WT animals was increased NF-KB translocation at 0.5 hours, which reached a significant level at 1 hour. In $T L R 4^{4^{--}}$mice, LPS failed to induce NF- $\mathrm{KB}$ translocation.

To address the role of NF- $\mathrm{\kappa B}$ in mediating LPS upregulation of TLR2, we determined the effects of IKK-NBD,

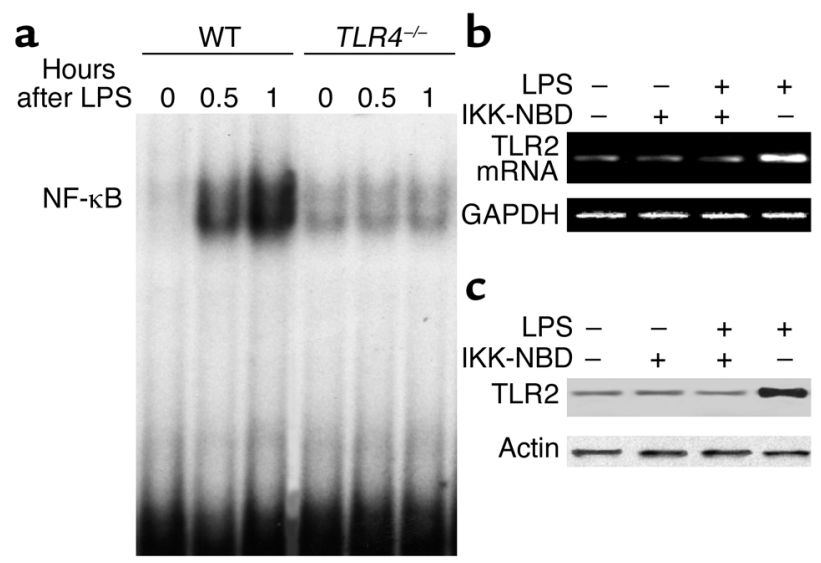

Figure 2

NF-KB mediates LPS-TLR4-induced TLR2 upregulation. (a) EMSA showing changes in NF-KB nuclear translocation in lung tissue of WT mice and TLR4 knockout mice after LPS intraperitoneal injection. (b and c) Confluent MLVECs were treated with LPS $(1 \mu \mathrm{g} / \mathrm{ml})$ in the presence or absence of the NF-KB inhibitor IKK-NBD $(100 \mu \mathrm{M})$ for 2 hours, followed by washing three times with HBSS and cell lysis. RT-PCR was performed using total RNA extracted from the cells (b), and Western blot was performed with the cell lysates $(\mathbf{c})$. The data are representative of three independent studies. 
a

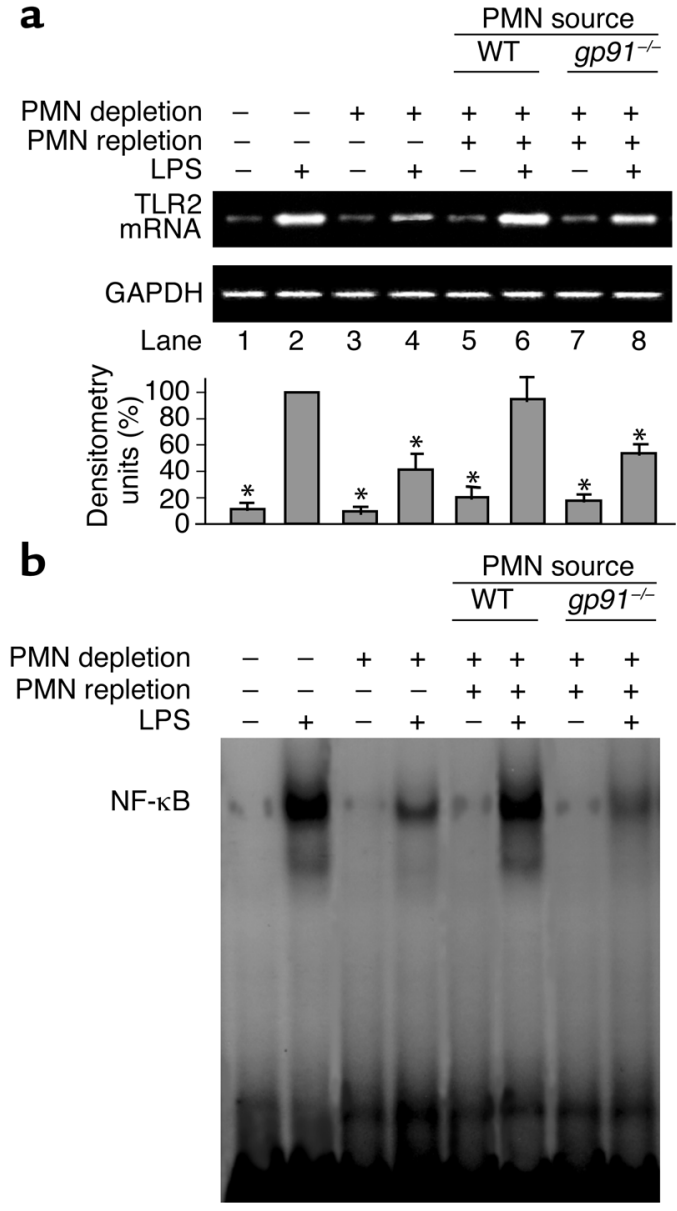

an NF- $\mathrm{BB}$ inhibitor (43), on the LPS-induced TLR2 expression in MLVECs. As shown in Figure 2, b and c, IKK-NBD $(100 \mu \mathrm{M})$ prevented the LPS upregulation of TLR2 mRNA and protein expression after LPS challenge.

PMNNADPH oxidase regulates LPS-induced TLR2 expression. As NF- $\mathrm{KB}$ signaling is oxidant-sensitive and PMN $\mathrm{NADPH}$ oxidase is a major source of superoxide, we hypothesized that PMN NADPH oxidase contributes to oxidant-dependent NF- $\mathrm{KB}$ activation. To determine the role of PMNs, we studied the responses to LPS challenge in lungs of WT mice following the depletion of circulating PMNs. In some cases, we repleted PMNs in mice made neutropenic to address the role in the response. As shown in Figure 3a, at 2 hours after LPS challenge, neutropenia in WT mice reduced TLR2 expression in lungs by about $60 \%$ (lane 4 ) compared with the control group treated with LPS alone (lane 2). Repletion with WT PMNs in these neutropenic WT mice restored the TLR2 expression in response to LPS (lane 6). However, repletion with PMNs derived from gp91pbox-l- mice failed to restore TLR2 expression (Figure $3 \mathrm{a}$, lane 8). These results demonstrate the critical role of PMN NADPH oxidase in the mechanism of LPS-induced TLR2 expression.

Figure $3 \mathrm{~b}$ shows the effects of PMN depletion and repletion on NF- $\mathrm{KB}$ translocation in lung tissue at 1 hour after LPS challenge. In WT animals, LPS caused a

\section{Figure 3}

Neutrophil NADPH oxidase signals LPS-induced TLR2 expression in lungs. (a) RT-PCR showing the effects of PMN depletion and repletion on the expression of TLR2 mRNA in lung tissue of WT mice 2 hours after intraperitoneal LPS. PMN depletion was performed with rabbit anti-mouse neutrophil serum $(150 \mu \mathrm{l}$ administered intraperitoneally) 16 hours before injection of LPS. PMN repletion was performed in neutropenic mice using $1 \times 10^{6} \mathrm{PMNs}$ isolated from whole blood of WT or gp91 phox-/- mice; cells in $150 \mu$ l of saline were injected into the tail vein. GAPDH mRNA was detected for normalizing the densitometry of TLR2. The graph depicts the mean and SEM from three mice. ${ }^{*} P<0.01$ compared with LPS treatment alone (lane 2). (b) EMSA showing the effect of PMN depletion and repletion on NF-KB nuclear translocation in lungs. PMN depletion and repletion experiments are as described in $\mathbf{a}$; the data are representative of three independent studies.

significant increase in NF- $\mathrm{KB}$ (lane 2), whereas in the neutropenic WT group, LPS-induced NF- $\mathrm{KB}$ was markedly reduced (lane 4). Repletion with WT PMNs restored the increase in NF- $\mathrm{KB}$ (lane 6). In contrast, repletion with $g p^{9} 1^{\text {phox- }- \text { - }}$ PMNs failed to restore NF- $\mathrm{KB}$ translocation in response to LPS (lane 8) compared with the LPS-alone group (lane 2). Thus, the results in Figure 3 show that the PMN NADPH oxidase complex signals LPS-induced TLR2 upregulation through the activation of NF- $\mathrm{KB}$.

Augmented LPS induction of TLR2 in endothelial cells requires PMN NADPH oxidase and PMN adhesion to endothelial cells. We next used the endothelial cell/PMN coculture system to address the question of whether the PMN NADPH oxidase complex induces TLR2 in endothelial cells and the requirement for PMN adhesion in the response. We used MLVECs in which we measured TLR 2 expression after LPS stimulation. LPS induced an increase in TLR2 mRNA and protein expression at 2 hours in WT MLVECs (Figure 4, a and b, lane 3). The coculture of MLVECs with WT PMNs markedly augmented TLR 2 expression in response to LPS (Figure 4, a and b, lane 4).

To address the role of endogenous endothelial NADPH oxidase in the regulation of TLR2 expression, we stimulated $p 47^{p b o x-1-}$ MLVECs with LPS. As shown in Figure 4c, LPS induced a gradual increase in TLR2 expression during the 2 - to 4 -hour period in $p 47^{p h o x-}-$ MLVECs. The level of TLR2 expression in $p 47^{p h o x-/-}$ MLVECs was less than that in WT MLVECs, indicating that endogenous endothelial NADPH oxidase is also involved in regulating LPS-induced TLR2 expression. However, coculture of MLVECs with WT PMNs caused a rapid and augmented TLR2 expression in response to LPS in both $p 47^{\text {phox- }- \text { - }}$ and WT groups, indicating the dominant role of PMN NADPH oxidase in the response (Figure 4c).

To further address the role of PMN-derived oxidants in signaling LPS-induced TLR2 expression in endothelial cells, membrane-permeable PEG-catalase $(1,000 \mathrm{U} / \mathrm{ml})$ was applied to the coculture system. As shown in Figure 4c, PEG-catalase attenuated the effect of PMNs in increasing TLR2 expression in LPS-stimulated MLVECs. 
The above data in mouse models and endothelial/ PMN coculture experiments show that oxidants derived from PMNs are critical in signaling LPSinduced NF- $\mathrm{B}$ activation and TLR2 expression in endothelial cells. An important question is whether PMN adhesion to vascular endothelial cells is required for PMNs to transmit the oxidant signals to endothelial cells. To determine the role of adhesive interactions, we studied the effects of anti-CD18 antibody (Santa Cruz Biotechnology Inc.). As shown in Figure 4, a and b, at 2 hours after LPS treatment, anti-CD18 antibody significantly reduced TLR2 expression in MLVECs (lane 6). Thus, the augmented expression of TLR2 in endothelial cells induced by PMNs requires an adhesive interaction mediated by CD18.

Increased TLR2 expression results in enhanced ICAM-1 expression and PMN migration. To address the physiological relevance of LPS/TLR4 activation of TLR2 expression in pulmonary vascular endothelial cells, we assessed ICAM-1 expression in lungs using sequential challenges of LPS and PGN, a ligand for TLR2. Injection of LPS at time 0 followed by a saline injection (control injection) at 2 hours caused a marked increase in ICAM-1 expression by 2 hours after LPS, a further increase at 4 hours, and a return toward basal level by 8 hours after LPS (Figure 5a). Saline injection at time 0 and PGN injection $(10 \mu \mathrm{g} / 10 \mathrm{~g}$ body wt intraperitoneally) at 2 hours caused a small increase in ICAM-1 expression after the PGN injection. However, the sequential injection of LPS at time 0 and PGN injection at 2 hours (at a time when TLR2 was upregulated) caused augmented and prolonged ICAM-1 expression compared with the single LPS challenge or sequential LPS challenge at time 0 and 2 hours (Figure $5 \mathrm{a}$ ). In contrast, the sequential injection of PGN at 2 hours after LPS failed to induce increased or stable ICAM-1 expression in TLR2 ${ }^{-/}$mice compared with WT mice (Figure $5 a)$. The sequential injection of LPS at 2 hours after the first dose of LPS did not alter the ICAM-1 expression pattern compared with the single LPS challenge; this observation might be the result of the downregulation of TLR4 in response to the first dose of LPS (data not shown). Administering either a single dose of PGN (at time 0 or 2 hours, respectively) or LPS together with PGN at time 0 also failed to enhance and prolong the ICAM-1 expression (Figure 5a).

To further address the role of TLR 2 in regulating ICAM-1 expression in response to Gram-negative infection, we measured lung ICAM-1 expression 4 hours after E. coli intraperitoneal challenge in WT and $T L R 2^{-/-}$mice, respectively. As shown in Figure $5 \mathrm{~b}$, E. coli induced less ICAM-1 expression in TLR2-/mouse lungs than in WT mouse lungs. The above data show an important role of the upregulated TLR2 in the amplification of ICAM-1 expression.

As ICAM-1-dependent PMN adhesion to endothelial cells is an important determinant of PMN migration, we also addressed the role of LPS-TLR4 upregulation of TLR2 in regulating PMN migration using the air pouch model in WT, $g p^{9} 1^{\text {phox-/- }}$, and TLR4 $4^{-/-}$mice. We first challenged the mice intraperitoneally with LPS or saline, and the chemokine MIP-2 was then injected into the air pouch of mice at 1 hour after the first administration of LPS or saline. This was followed by a second injection of either LPS or PGN 1 hour later. The pouch lavage fluid was collected for PMN counts at 2 hours and 8 hours after the first injection of LPS or saline. As shown in Figure 6a, MIP-2 induced basal PMN migration in WT, gp91phox-/-, and TLR $4^{-/}$mice at 2 hours and 8 hours after the saline injections (group 1 and group 3). At 2 hours after LPS injection, MIP-2 caused a 2.4-fold increase in PMN migration in WT and gp91 phox-/- mice (group 2) compared with the saline control (group 1), but failed to increase PMN migration in TLR4 $4^{-/-}$mice. At the 8hour timepoint, the LPS/saline challenge protocol caused 2.7- and 2.2-fold increases in PMN migration in WT and gp91 phox-/- mice (group 5) compared with the saline control (group 3), respectively, in response

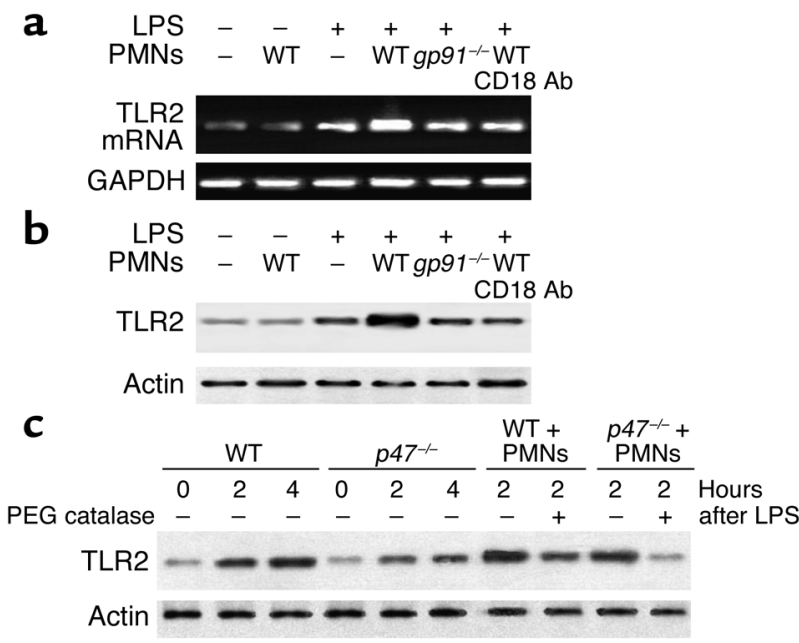

\section{Figure 4}

Augmented LPS induction of TLR2 in endothelial cells requires PMN $\mathrm{NADPH}$ oxidase and PMN adhesion. Studies determined the effects of PMNs cocultured with MLVECs on MLVEC TLR2 expression in response to LPS. MLVECs were isolated and cultured as described in Methods and treated with LPS $(1 \mu \mathrm{g} / \mathrm{ml})$ for 2 hours. The cocultured PMNs were isolated from WT and $g p 91$ phox $-1-$ mice, respectively, and were added at a concentration of $1 \times 10^{5} \mathrm{cell} / \mathrm{s} / \mathrm{ml}$. At the end of incubation with LPS, MLVECs were washed with HBSS three times and then lysed with lysis buffer. Cell lysates were subjected to extraction of total RNA and RT-PCR analysis (a) as well as Western blotting with anti-TLR2 antibody (b). To address the effects of CD18 on PMN-activated TLR2 expression in MLVECs, confluent MLVECs were treated with LPS in the presence of WT PMNs and anti-CD18 antibody for 2 hours, followed by washing with HBSS three times. This was followed by RT-PCR and Western analysis ( $\mathbf{a}$ and $\mathbf{b}$, lane 6 ). (c) To address the role of endogenous endothelial NADPH oxidase in the regulation of TLR2 expression, MLVECs isolated from $p 47^{\text {phox }-/-}$ mice were stimulated with LPS and cocultured with or without WT PMNs for the times indicated. To address the role of oxidants derived from PMNs in LPSinduced TLR2 expression in endothelial cells, membrane-permeable PEG-catalase $(1,000 \mathrm{U} / \mathrm{ml})$ was applied to the coculture system. The data are representative of three independent studies. 
a

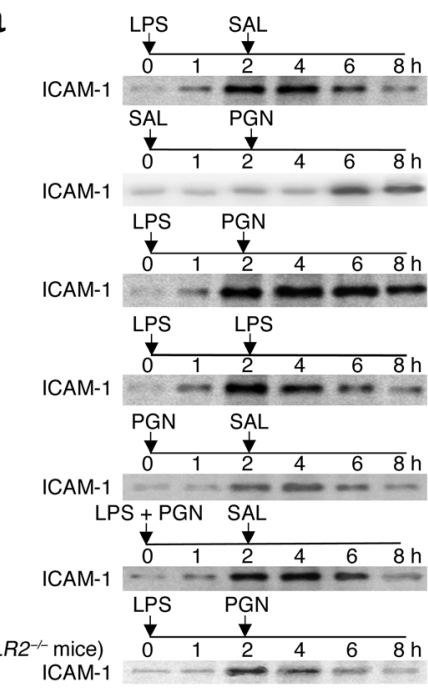

to MIP-2, but did not alter PMN migration in TLR4 $4^{-/-}$ mice compared with the saline group. Sequential double LPS challenge increased PMN migration to 2.8and 2.4-fold in WT and gp91phox-/- mice, respectively, in response to MIP-2, but again did not increase PMN migration in TLR4 $4^{-/-}$mice (group 6). Sequential injection of PGN at 2 hours after the initial LPS injection, however, markedly increased PMN migration in WT mice, by 4.1-fold (group 7) compared with the saline group. In contrast, the sequential challenge of LPS and PGN did not significantly increase PMN migration in gp $91^{\text {phox-/- }}$ and TLR4 $4^{-/-}$mice (group 7) compared with either LPS/saline (group 5) or sequential double LPS challenge (group 6). Injection of PGN at 2 hours after saline caused a small (statistically insignificant) increase in PMN migration in WT, $g p^{91^{\text {phox-l-}} \text {, and }}$ TLR4 $4^{-/}$mice (group 4) compared with the saline group (group 3, Figure 6a).

\section{Figure 6}

Effects of sequential challenges of LPS and PGN on MIP-2-induced PMN migration in vivo and transalveolar PMN migration. (a) Data obtained using in vivo air pouch model in which MIP-2 was used to

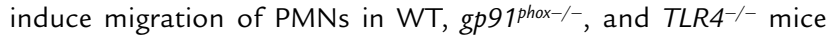
sequentially challenged with intraperitoneal injections of two doses of LPS or LPS followed by PGN (as described in Methods). Sequential double LPS challenge increased PMN migration to 2.8- and 2.4-fold in WT and gp91 phox- - - mice, respectively, in response to MIP-2, but did not increase PMN migration in TLR4 $4^{-/-}$mice. Sequential injection of PGN 2 hours after LPS, however, markedly increased PMN migration in WT mice, to 4.1-fold that measured in the saline group. In contrast, sequential treatment of LPS and PGN did not significantly increase PMN migration in gp91 phox-/- or TLR4 $4^{-/-}$mice compared with either single LPS or sequential double LPS groups. ${ }^{*} P<0.01$ compared with WT animals within the same treatment group; ${ }^{*} P<0.01$ compared with WT animals in other treatment groups ( $n=3$ per group). Numbers (1-7, top) indicate the different groups. (b) To address the role of TLR2 in regulating transalveolar PMN migration in the lung, E. coli was injected intraperitoneally $\left(1 \times 10^{5}\right.$ cells $/ 10 \mathrm{~g}$ body wt $)$ into WT and TLR2 $2^{-/}$mice, and BALF PMNs were counted 4 hours later $(n=3$ per group). White bars, saline alone; gray bars, E. coli.

\section{Figure 5}

Increased TLR2 expression results in augmented and stable ICAM-1 expression. (a) The results show the effects of sequential challenges of LPS and PGN, the ligands for TLR4 and TLR2, respectively, on ICAM-1 expression in lungs. In WT mice, LPS (10 $\mu \mathrm{g} / 10 \mathrm{~g}$ body $w \mathrm{t}), \mathrm{PGN}(10$ $\mu \mathrm{g} / 10 \mathrm{~g}$ body $\mathrm{wt}$ ), LPS plus PGN, or saline (SAL) was injected intraperitoneally at time 0 , and 2 hours later, a dose of either LPS $(10 \mu \mathrm{g} / 10 \mathrm{~g}$ body wt), PGN (10 $\mu \mathrm{g} / 10 \mathrm{~g}$ body $w \mathrm{w})$, or saline was injected. Lung tissue was harvested at the times indicated, and ICAM-1 protein was detected using Western blotting. (b) WT or TLR2 ${ }^{-1-}$ mice were injected with E. coli $\left(1 \times 10^{5}\right.$ cells/10 g body wt, intraperitoneally $)$ and ICAM-1 expression in the lung was measured 4 hours later by Western blotting. The data are representative of three independent studies.

To further address the role of TLR 2 in regulating PMN migration in lungs, WT and TLR2 $2^{-/}$mice were challenged with E. coli and PMNs were counted in the bronchoalveolar lavage fluid (BALF). E. coli caused a 42.5 -fold increase in BALF PMNs in WT mice compared with saline-treated control, but only a 17.5 -fold increase in BALF PMNs in TLR2 $2^{-/}$mice, indicating an important role of TLR2 in regulating PMN migration in response to Gram-negative infection (Figure 6b). Taken together, these data show the important role of TLR4 signaling and the PMN NADPH oxidase system in mediating the augmented and stable PMN migration response secondary to the upregulation of TLR2 expression.

\section{Discussion}

We observed that LPS-TLR4 signaling upregulated TLR2 expression in endothelial cells, and PMN NADPH oxidase-derived oxidants played a critical role in the mechanism of TLR2 upregulation. This cross talk between TLR 4 and TLR2 in endothelial cells resulted in the amplification and stable expression of ICAM- 1 in response to the bacterial products LPS and PGN, which
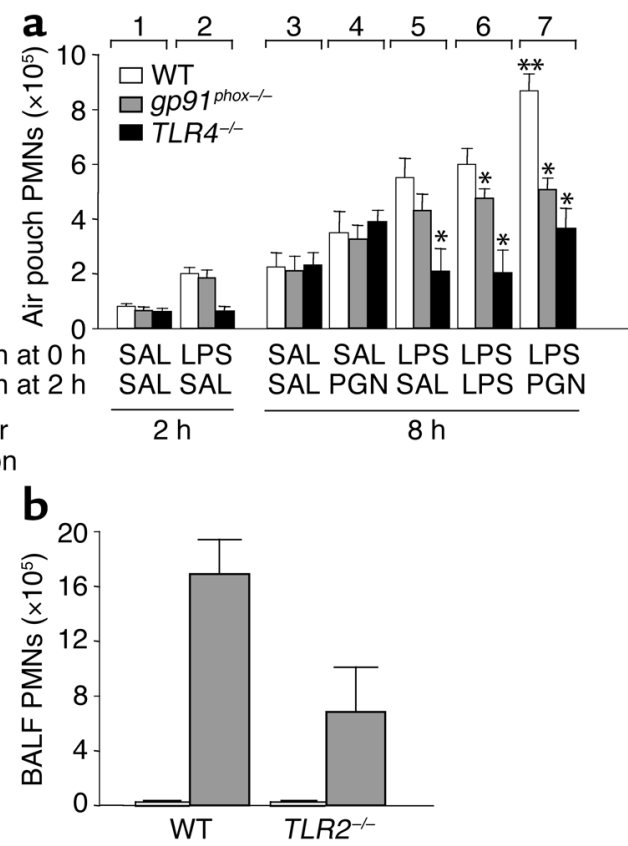


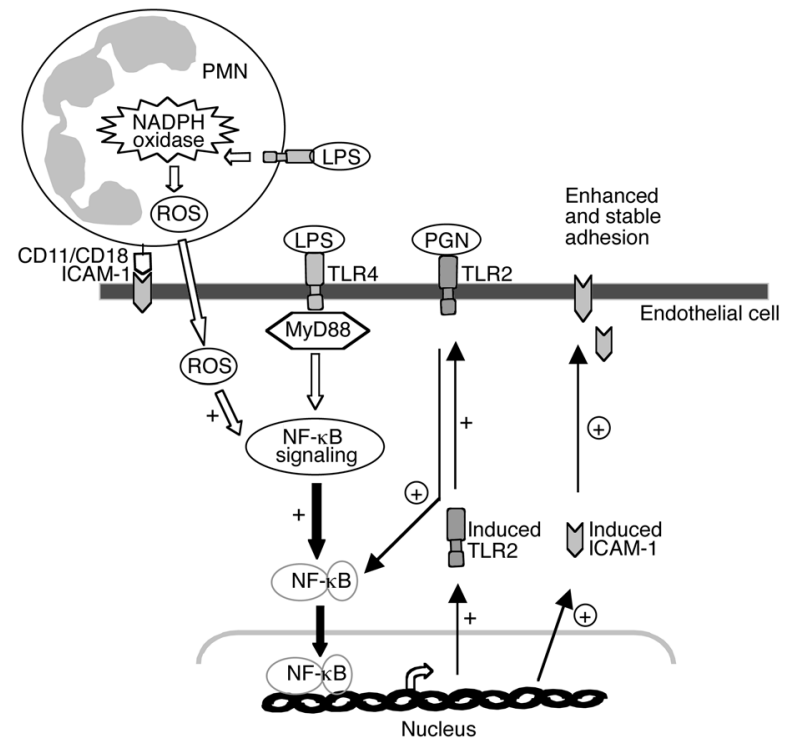

Figure 7

Model of PMN NADPH oxidase-derived oxidant signaling in mediating the TLR4-TLR2 cross talk in endothelial cells. LPS stimulation induces NADPH oxidase activation and production of reactive oxygen species (ROS) in PMNs as well as the initiation of MyD88dependent NF- $\mathrm{KB}$ signaling in endothelial cells and the consequent expression of TLR2 and ICAM-1. Adhesion of PMNs to endothelial cells is mediated by binding of constitutive ICAM-1 to CD18 integrin and provides the appropriate coupling required for $\mathrm{PMNs}$ to transmit oxidant signals to endothelial cells. The oxidants augment NF- $\kappa B$ signaling and TLR2 expression (+), which results in the augmented response of the cell to PGN, thereby amplifying ICAM-1 expression (circled + ) and promoting stable adhesion of PMNs to endothelial cells and increased PMN migration. Thus, the PMN NADPH oxidase-mediated TLR4-TLR2 cross talk activates a positive feedback signal leading to sustained and amplified endothelial activation in response to invading pathogens.

activate TLR4 and TLR2, respectively. Thus, the present study demonstrates a novel function of the phagocytic cell NADPH oxidase complex in the innate immunity mediated by TLRs; that is, PMN NADPH oxidase regulates the TLR 2 expression induced by TLR4, which by signaling the expression of ICAM-1 in endothelial cells controls the transendothelial PMN migration response to sites of infection (Figure 7).

The role of TLR 4 signaling in regulating TLR 2 expression was clearly delineated in the studies using TLR4 $4^{--}$ mice. We showed that LPS challenge in WT mice induced the upregulation of TLR2, whereas this response was impaired in TLR4 $4^{-/}$mice. We also showed that MyD88 mediated the TLR4-TLR2 cross talk, since LPS challenge of $M y D 88^{-/}$mice failed to induce increased TLR 2 expression. The phylogenetically conserved innate immunity signaling pathways activated by TLRs in mammals can induce responses through the transcription factor NF-KB (17). In both in vivo and in vitro studies, we observed the activation of NF- $\mathrm{\kappa B}$ in association with increased expression of TLR 2 after LPS challenge and reduced expression of TLR2 in endothelial cells in which NF-кB was inhibited by IKK-NBD.
Thus, these results demonstrate the essential role of NF-KB in mediating the TLR4-TLR2 cross talk.

The NADPH oxidase complex in phagocytic cells and the generation of oxidants serve a crucial function in host defense against invading microorganisms (29). $\mathrm{NADPH}$ oxidase found in endothelial cells can also generate oxidants, although its capacity is limited compared to NADPH oxidase in phagocytic cells (29). We demonstrated in mouse experiments that (a) PMN depletion in WT mice reduced LPS-activated TLR2 expression (b) repletion of WT mice with WT PMNs restored TLR2 expression in response to LPS challenge, and importantly (c) repletion of WT animals with gp91phox-/- PMNs failed to restore TLR2 expression in response to LPS challenge. These data show that PMN NADPH oxidase is an important determinant of LPSinduced NF- $\mathrm{KB}$ activation and the consequent TLR2 expression. Our findings are in accord with the described role of NADPH oxidase in the mechanism of NF- $\kappa B$ activation induced by alcohol in the liver (44) and surfactant $\mathrm{D}$ in alveolar macrophages (45), and in the expression of ICAM-1 in endothelial cells in response to TNF- $\alpha$ (26).

Since the regulation of TLR2 assessed in lung tissue could be the result of TLR 2 expression in multiple cell types (12-16), studies were also made in a coculture system of mouse MLVECs and PMNs to address the specific role of PMN NADPH oxidase in signaling TLR2 expression in endothelial cells. As shown in Figure 4a, WT PMNs resulted in augmented TLR2 expression in MLVECs, indicating that direct PMN-endothelial cell interaction mediated the response. Addition of the antioxidant PEG-catalase to the coculture prevented the response, indicating that oxidant signaling was responsible for the TLR2 expression. Thus, endothelial cells are important targets for PMN NADPH oxidase-derived oxidants, and thereby mediate NF-KB activation and TLR2 expression in endothelial cells. Transcellular cross talk between PMNs and endothelial cells is required for the TLR4-induced upregulation of TLR2, which thus promotes the enhanced activation of endothelial cells in response to bacterial components.

We observed that LPS induced TLR2 expression even in PMN-depleted WT mice (Figure 3a). We also observed that MLVECs from $p 47^{\text {bhox }-/-}$ mice had a lower expression of TLR2 in response to LPS than did WT MLVECs. These observations are consistent with the finding that LPS is capable of activating TLR2 expression in human endothelial cells in an NF-KB-dependent manner (20). Endogenous NADPH oxidase in endothelial cells is also believed to be involved in signal transduction (29). However, TLR2 expression in the presence of WT PMNs was markedly augmented in response to LPS. Moreover, coculturing of MLVECs from $p 47^{p h o x-/-}$ mice with WT PMNs resulted in the same increased expression of TLR2

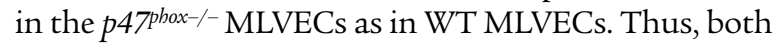
in vivo and cell culture studies point to the oxidants derived from PMN NADPH oxidase as playing the dominant role in inducing TLR2 expression. 
It has been reported that CD11/CD18 integrins mediate PMN oxidant production $(46,47)$. The present results showed that the CD18-dependent adhesion of PMNs to endothelial cells is required for the PMN NADPH oxidase-dependent expression of TLR2 in endothelial cells. The anti-CD18 monoclonal antibody markedly reduced TLR2 expression in endothelial cells as induced by PMNs. Thus, CD18 may contribute not only to PMN oxidant production, but to PMN adhesion to endothelial cells by binding to ICAM-1, and thereby provides the necessary cell interaction for PMNs to transmit oxidant signals to endothelial cells. Although ICAM-1 is expressed constitutively in endothelial cells, its level increases in response to proinflammatory stimuli (26). The present study suggests that some degree of early PMN recruitment dependent on CD18 binding to the constitutively expressed ICAM-1 is necessary for the subsequent enhanced and stable ICAM-1 expression.

Previous studies showed a role of TLR2 in LPS signaling $(15,48)$; however, other studies have shown that human or murine TLR2 was not involved in LPS signaling $(18,49-51)$ and that highly purified LPS failed to directly activate cells through TLR2 (38). To address the role of TLR4 signaling in upregulation of TLR2 expression, we used repurified $E$. coli LPS to eliminate any possible artifacts of non-LPS contamination. We observed that the upregulation of TLR2 was secondary to the activation of TLR4 signaling rather than direct LPS activation of TLR 2 .

TLR2 mediates cellular responses to a wide variety of infectious pathogens and their products. These include yeast cell walls, whole mycobacteria, mycobacterial lipoarabinomannan, whole Gram-positive bacteria, Treponema glycolipid, Trypanosoma cruzi glycophosphatidylinositol anchor, and PGN $(8,9,11,52-56)$. For TLR2 activity, the ligand specificity as well as signaltransducing ability are determined by heterodimeric interactions with other TLRs, such as TLR6 and TLR1 (57). Dimerization of the cytoplasmic domain of TLR2 did not induce cytokine production in a macrophage cell line, whereas the cytoplasmic portion of TLR2 can functionally pair with TLR6 or TLR1, resulting in cytokine production. Results from expression of dominant-negative TLR2 or dominant-negative TLR6 in a macrophage cell line showed that TLR6 and TLR2 functioned cooperatively to detect Gram-positive bacteria, PGN, and zymosan, whereas TLR2 functioned either alone or with TLRs (other than TLR6) to detect bacterial lipopeptides (58). However, it has also been reported that responses to PGN were not abolished in $\mathrm{TLR6}^{-/-}$mice (59). This discrepancy may be the result of artifacts associated with overexpression of proteins and to possible impurities in the microbial components used. Nevertheless, it is clear that TLR2 can recognize and transduce signaling of PGN, the thick rigid layer found in both Gram-positive and Gram-negative cells. In Gram-positive bacteria, PGN accounts for 50\% of cell weight and $90 \%$ of cell wall weight, whereas in
Gram-negative bacteria, the PGN layer is much thinner, comprising only $15-20 \%$ of cell wall weight.

However, the fundamental question remains whether TLR4-TLR2 cross talk amplifies the endothelial cell activation response to invading pathogens. We addressed this question, which is important for understanding the innate immune function of the endothelium. In the present study, following the sequential LPS and PGN challenge protocol in WT mice, we observed that the LPS-TLR4 upregulation of TLR2 resulted in marked augmentation of ICAM-1 expression in lungs in response to PGN. This response was significantly reduced in lungs of TLR2-/- mice. In addition, in studies using the air pouch method to determine PMN migration, the sequential challenge with LPS and PGN caused a marked increase in PMN migration in WT mice, but this did not occur in gp91 phox-/- or $\mathrm{TLR}^{\mathrm{L} 4^{-/}}$mice, or following either single LPS or sequential double LPS challenge. We observed a persistent small increase in PMN migration in $T L R 4^{-1-}$ mice after the sequential challenge with LPS and PGN (Figure 6a). This TLR4-independent increase in PMN migration may be caused by PGN through activation of TLR2, because neither saline nor LPS increased PMN migration in the TLR4 $4^{-/}$mice. Also, this level of PMN migration was equivalent to that seen in saline-PGN-treated WT animals. Further, our data showed that genetic deficiency of TLR2 caused impaired alveolar PMN infiltration in response to Gram-negative bacteria. Taken together, these data demonstrate the important role of the PMN NADPH oxidase system and TLR4 signaling in regulating the augmented and stable $\mathrm{PMN}$ migration response through the upregulation of TLR2 expression.

In summary, the present study identifies a previously unrecognized function of PMN NADPH oxidase-derived oxidant signaling in mediating TLR4TLR2 cross talk. PMN NADPH oxidase activates transcellular oxidant signaling through its ability to induce NF- $\mathrm{KB}$ activation in endothelial cells. Although endogenous NADPH oxidase in endothelial cells can induce oxidant signaling, and endothelial TLR4 may be directly activated by LPS (60), the activation of PMN NADPH markedly amplifies the endothelial response. Thus, the endothelial cell is an important target for PMN NADPH oxidase activity, resulting in augmented TLR2 expression and stable endothelial expression of ICAM-1, which acts in a positive feedback manner to amplify the host response to invading pathogens (Figure 7 ).

\section{Acknowledgments}

This work was supported by NIH grants T32 HL07829, HL-45638, HL-60678, and HL-27016.

\footnotetext{
1. Mantovani, A., Bussolino, F., and Dejana, E. 1992. Cytokine regulation of endothelial cell function. FASEB J. 6:2591-2599.

2. Daniel, T.O., and Abrahamson, D. 2000. Endothelial signal integration in vascular assembly. Annu. Rev. Physiol. 62:649-671.

3. Iivanainen, E., Kahari, V.M., Heino, J., and Elenius, K. 2003. Endothelial cell-matrix interactions. Microsc. Res. Tech. 60:13-22.
} 
4. Zhang, P., Summer, W.R., Bagby, G.J., and Nelson, S. 2000. Innate immunity and pulmonary host defense. Immunol. Rev. 173:39-51.

5. Pober, J.S., and Cotran, R.S. 1990. The role of endothelial cells in inflammation. Transplantation. 50:537-544.

6. Pober, J.S., and Cotran, R.S. 1990. Cytokines and endothelial cell biology. Physiol. Rev. 70:427-451.

7. Ingber, D.E. 2002. Mechanical signaling and the cellular response to extracellular matrix in angiogenesis and cardiovascular physiology. Circ. Res. 91:877-887.

8. Lien, E., et al. 1999. Toll-like receptor 2 functions as a pattern recognition receptor for diverse bacterial products. J. Biol. Chem. 274:33419-33425.

9. Schwandner, R., Dziarski, R., Wesche, H., Rothe, M., and Kirschning, C.J. 1999. Peptidoglycan- and lipoteichoic acid-induced cell activation is mediated by toll-like receptor 2. J. Biol. Chem. 274:17406-17409.

10. Underhill, D.M., Ozinsky, A., Smith, K.D., and Aderem, A. 1999. Toll-like receptor-2 mediates mycobacteria-induced proinflammatory signaling in macrophages. Proc. Natl. Acad. Sci. U. S. A. 96:14459-14463.

11. Yoshimura, A., et al. 1999. Cutting edge: recognition of Gram-positive bacterial cell wall components by the innate immune system occurs via Toll-like receptor 2. J. Immunol. 163:1-5.

12. Flo, T.H., et al. 2001. Differential expression of Toll-like receptor 2 in human cells. J. Leukoc. Biol. 69:474-481.

13. Muzio, M., et al. 2000. Differential expression and regulation of toll-like receptors (TLR) in human leukocytes: selective expression of TLR3 in dendritic cells. J. Immunol. 164:5998-6004.

14. Visintin, A., et al. 2001. Regulation of Toll-like receptors in human monocytes and dendritic cells. J. Immunol. 166:249-255.

15. Yang, R.B., et al. 1998. Toll-like receptor- 2 mediates lipopolysaccharideinduced cellular signalling. Nature. 395:284-288.

16. Imler, J.L., and Hoffmann, J.A. 2001. Toll receptors in innate immunity. Trends Cell Biol. 11:304-311.

17. Medzhitov, R. 2001. Toll-like receptors and innate immunity. Nat. Rev. Immunol. 1:135-145.

18. Takeuchi, O., et al. 1999. Differential roles of TLR2 and TLR4 in recognition of gram-negative and gram-positive bacterial cell wall components. Immunity. 11:443-451.

19. Fan, J., and Malik, A.B. 2003. Toll-like receptor-4 (TLR4) signaling augments chemokine-induced neutrophil migration by modulating cell surface expression of chemokine receptors. Nat. Med. 9:315-321.

20. Faure, E., et al. 2001. Bacterial lipopolysaccharide and IFN-gamma induce Toll-like receptor 2 and Toll-like receptor 4 expression in human endothelial cells: role of NF-kappa B activation. J. Immunol. 166:2018-2024.

21. Ashkenazi, A., and Dixit, V.M. 1998. Death receptors: signaling and modulation. Science. 281:1305-1308.

22. Buttke, T.M., and Sandstrom, P.A. 1994. Oxidative stress as a mediator of apoptosis. Immunol. Today. 15:7-10.

23. True, A.L., Rahman, A., and Malik, A.B. 2000. Activation of NF-kappaB induced by $\mathrm{H}(2) \mathrm{O}(2)$ and TNF-alpha and its effects on ICAM-1 expression in endothelial cells. Am. J. Physiol. Lung Cell. Mol. Physiol. 279:L302-L311.

24. Peng, Q., Wei, Z., and Lau, B.H. 2000. Pycnogenol inhibits tumor necrosis factor-alpha-induced nuclear factor kappa B activation and adhesion molecule expression in human vascular endothelial cells. Cell. Mol. Life Sci. 57:834-841.

25. Bowie, A., and O'Neill, L.A. 2000. Oxidative stress and nuclear factorkappaB activation: a reassessment of the evidence in the light of recent discoveries. Biochem. Pharmacol. 59:13-23.

26. Fan, J., Frey, R.S., Rahman, A., and Malik, A.B. 2002. Role of neutrophil NADPH oxidase in the mechanism of tumor necrosis factor-alphainduced NF-kappa B activation and intercellular adhesion molecule-1 expression in endothelial cells. J. Biol. Chem. 277:3404-3411.

27. Elbim, C., et al. 1999. Defective priming of the phagocyte oxidative burst in a child with recurrent intracellular infections. Microbes Infect. 1:581-587.

28. Simms, H.H., and D’Amico, R. 1994. Regulation of intracellular polymorphonuclear leukocyte $\mathrm{Fc}$ receptors by lipopolysaccharide. Cell. Immunol. 157:525-541.

29. Babior, B.M. 1999. NADPH oxidase: an update. Blood. 93:1464-1476.

30. Pollock, J.D., et al. 1995. Mouse model of X-linked chronic granulomatous disease, an inherited defect in phagocyte superoxide production. Nat. Genet. 9:202-209.

31. Jackson, S.H., Gallin, J.I., and Holland, S.M. 1995. The p47phox mouse knock-out model of chronic granulomatous disease. J. Exp. Med. 182:751-758.

32. Adachi, O., et al. 1998. Targeted disruption of the MyD88 gene results in loss of IL-1- and IL-18-mediated function. Immunity. 9:143-150.

33. Takeuchi, O., Hoshino, K., and Akira, S. 2000. Cutting edge: TLR2-defi- cient and MyD88-deficient mice are highly susceptible to Staphylococcus aureus infection. J. Immunol. 165:5392-5396.

34. Hoshino, K., et al. 1999. Cutting edge: Toll-like receptor 4 (TLR4)-deficient mice are hyporesponsive to lipopolysaccharide: evidence for TLR4 as the Lps gene product. J. Immunol. 162:3749-3752.

35. Szucs, S., Varga, C., Ember, I., and Kertai, P. 1994. The separation of the granulocytes from different rat strains. A comparative study. J. Immunol. Methods. 167:245-251.

36. Hohler, B., Holzapfel, B., and Kummer, W. 2000. NADPH oxidase subunits and superoxide production in porcine pulmonary artery endothelial cells. Histochem. Cell Biol. 114:29-37.

37. Deryckere, F., and Gannon, F. 1994. A one-hour minipreparation technique for extraction of DNA-binding proteins from animal tissues. Biotechniques. 16:405.

38. Hirschfeld, M., Ma, Y., Weis, J.H., Vogel, S.N., and Weis, J.J. 2000. Cutting edge: repurification of lipopolysaccharide eliminates signaling through both human and murine toll-like receptor 2. J. Immunol. 165:618-622.

39. Manthey, C.L., et al. 1994. Endotoxin-induced early gene expression in C3H/HeJ (Lpsd) macrophages. J. Immunol. 153:2653-2663.

40. Edwards, J.C., Sedgwick, A.D., and Willoughby, D.A. 1981. The formation of a structure with the features of synovial lining by subcutaneous injection of air: an in vivo tissue culture system. J. Pathol. 134:147-156.

41. Henneke, P., and Golenbock, D.T. 2001. TIRAP: how Toll receptors fraternize. Nat. Immunol. 2:828-830.

42. Musikacharoen, T., Matsuguchi, T., Kikuchi, T., and Yoshikai, Y. 2001. NF-kappa B and STAT5 play important roles in the regulation of mouse Toll-like receptor 2 gene expression. J. Immunol.166:4516-4524.

43. May, M.J., et al. 2000. Selective inhibition of NF-kappaB activation by a peptide that blocks the interaction of NEMO with the IkappaB kinase complex. Science. 289:1550-1554.

44. Kono, H., et al. 2000. NADPH oxidase-derived free radicals are key oxidants in alcohol-induced liver disease. J. Clin. Invest. 106:867-872.

45. Yoshida, M., Korfhagen, T.R., and Whitsett, J.A. 2001. Surfactant protein D regulates NF-kappaB and matrix metalloproteinase production in alveolar macrophages via oxidant-sensitive pathways. J. Immunol. 166:7514-7519.

46. Nathan, C., et al. 1989. Cytokine-induced respiratory burst of human neutrophils: dependence on extracellular matrix proteins and CD11/CD18 integrins. J. Cell Biol. 109:1341-1349.

47. Shappell, S.B., et al. 1990. Mac-1 (CD11b/CD18) mediates adherencedependent hydrogen peroxide production by human and canine neutrophils. J. Immunol. 144:2702-2711.

48. Kirschning, C.J., Wesche, H., Merrill Ayres, T., and Rothe, M. 1998. Human toll-like receptor 2 confers responsiveness to bacterial lipopolysaccharide. J. Exp. Med. 188:2091-2097.

49. Heine, H., et al. 1999. Cutting edge: cells that carry A null allele for tolllike receptor 2 are capable of responding to endotoxin. J. Immunol. 162:6971-6975

50. Underhill, D.M., et al. 1999. The Toll-like receptor 2 is recruited to macrophage phagosomes and discriminates between pathogens. Nature. 401:811-815

51. Faure, E., et al. 2000. Bacterial lipopolysaccharide activates NF-kappaB through toll-like receptor 4 (TLR-4) in cultured human dermal endothelial cells. Differential expression of TLR-4 and TLR-2 in endothelial cells. J. Biol. Chem. 275:11058-11063.

52. Means, T.K., et al. 1999. Human toll-like receptors mediate cellular activation by Mycobacterium tuberculosis. J. Immunol. 163:3920-3927.

53. Flo, T.H., et al. 2000. Human toll-like receptor 2 mediates monocyte activation by Listeria monocytogenes, but not by group B streptococci or lipopolysaccharide. J. Immunol. 164:2064-2069.

54. Takeuchi, O., et al. 2000. Cellular responses to bacterial cell wall components are mediated through MyD88-dependent signaling cascades. Int. Immunol. 12:113-117.

55. Opitz, B., et al. 2001. Toll-like receptor-2 mediates Treponema glycolipid and lipoteichoic acid-induced NF-kappaB translocation. J. Biol. Chem. 276:22041-22047.

56. Campos, M.A., et al. 2001. Activation of Toll-like receptor-2 by glycosylphosphatidylinositol anchors from a protozoan parasite. J. Immunol. 167:416-423.

57. Ozinsky, A., et al. 2000. The repertoire for pattern recognition of pathogens by the innate immune system is defined by cooperation between toll-like receptors. Proc. Natl. Acad. Sci. U. S. A. 97:13766-13771.

58. Akira, S., Takeda, K., and Kaisho, T. 2001. Toll-like receptors: critical proteins linking innate and acquired immunity. Nat. Immunol. 2:675-680.

59. Takeuchi, O., et al. 2001. Discrimination of bacterial lipoproteins by Toll-like receptor 6. Int. Immunol. 13:933-940.

60. Andonegui, G., et al. 2003. Endothelium-derived Toll-like receptor-4 is the key molecule in LPS-induced neutrophil sequestration into lungs. J. Clin. Invest. 111:1011-1020. doi:10.1172/JCI200316510. 